\title{
Clinicoradiological and pathological correlations in patients with solitary cysticercus granuloma and epilepsy: focus on presence of the parasite and oedema formation
}

\author{
Vedantam Rajshekhar, Geeta Chacko, R P Haran, Mathew J Chandy, Sushil M Chandi
}

\begin{abstract}
A study of the clinical, radiological, and pathological correlations in 43 patients with solitary cysticercus granuloma and epilepsy focused on factors that might help in predicting the presence of the parasite in the granuloma and those that might influence the formation of oedema around the granuloma. The duration of symptoms (<six months and $\geqslant$ six months) and CT morphology of the granuloma (ring and disc, type A; nodular lesion, type B) were studied as factors that could possibly predict the presence of the parasite in the granuloma. The influence of sex of the patient and the presence of a neutrophilic response in the granuloma on the intensity of oedema around the lesion as seen on CT was also studied. The pathological features were studied in the excised granulomas. The intact or degenerated form of the cysticercus was evident in 22 of 43 specimens. Neither the duration of seizures $(P=0.17)$ nor the type of lesion on CT $(P=0 \cdot 16)$ was predictive of the presence of the parasite in the granuloma. The sex of the patient $(P=0.51)$ and the neutrophilic response in the specimen $(P=0.73)$ did not correlate with the degree of oedema on CT indicating that neither of these host factors was a major determinant of oedema production. The findings point to the varied and unpredictable natural history of solitary cysticercus granulomas and the complex nature of host-parasite interactions in individual patients. The inability to predict the presence of the parasite in the granuloma on the basis of the clinical or radiological features precludes a selection of patients with such lesions for cysticidal drug treatment.
\end{abstract}

(F Neurol Neurosurg Psychiatry 1995;59:284-286)

Neurological Sciences and Pathology, Cinristian Medical College and Hospital, Vellore, India V Rajshekhar G Chacko R P Haran $M$ J Chandy S M Chandi

Correspondence to: Dr V Rajshekhar, Department of Neurological Sciences, Christian Medical College and Hospital, Vellore 632004, India. Received 19 October 1994 and in revised form 22 March 1995

Accepted 16 May 1995

Keywords: cysticercus granuloma; computerised tomography; oedema; epilepsy

Solitary cysticercus granulomas seen as solitary, small $(<20 \mathrm{~mm})$, enhancing lesions on CT are a common cause of epilepsy in many parts of the world. ${ }^{1}$ Their CT morphology, biological behaviour, and clinical manifestations have recently been elucidated. ${ }^{1-4}$ Epilepsy is their commonest manifestation and they rarely, if ever, cause a progressive focal neurological deficit or raised intracranial pressure. ${ }^{3}$ These lesions usually resolve spontaneously but an appreciable number persist for prolonged periods ranging from many months to years. Cysticidal drug treatment has been used to hasten the resolution of these lesions, but has not been universally effective. ${ }^{5}$ The factors affecting the response of a granuloma to cysticidal drugs are not clear.

The parasite is often not visualised in granulomas which are excised. With a view to predicting the presence of the parasite in the granuloma and hence possibly selecting patients for cysticidal drug treatment, we attempted to determine whether there were any correlations between the clinicoradiological features and the presence of a parasite in the excised granuloma. The effect of sex of the patient and neutrophilic response in the granuloma was also compared with the degree of perilesional oedema.

\section{Materials and methods}

PATIENT POPULATION

The study was on 43 unselected patients (27 male and 16 female) who presented with seizures and a single, small $(<20 \mathrm{~mm})$, enhancing lesion on CT. The granuloma was excised totally in all the patients after a CT guided stereotactic craniotomy. The indications for excision of the granuloma were (a) for histological diagnosis; patients who underwent surgery for this indication presented in the initial period of the study (1987 to 1991) when the management of these lesions was still being debated. At that time their aetiology was unclear and antituberculous treatment was considered an appropriate alternative to non-specific treatment; and (b) for epilepsy not easily controlled with drugs. The patients' ages ranged from 2-47 (median 27) years. Twenty three patients had had symptoms for less than six months ( 0.5 to six months; median two months) whereas the others (20 patients) had had symptoms for periods ranging from eight months to 22 years 


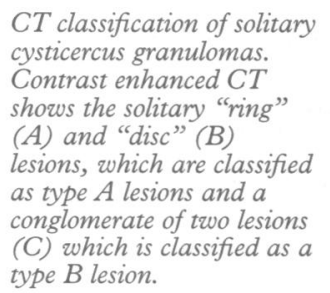

CT classification of solitary cysticercus granulomas. Contrast enhanced CT shows the solitary "ring" (A) and "disc" (B) lesions, which are classified as type $A$ lesions and a conglomerate of two lesions (C) which is classified as a type B lesion.

A
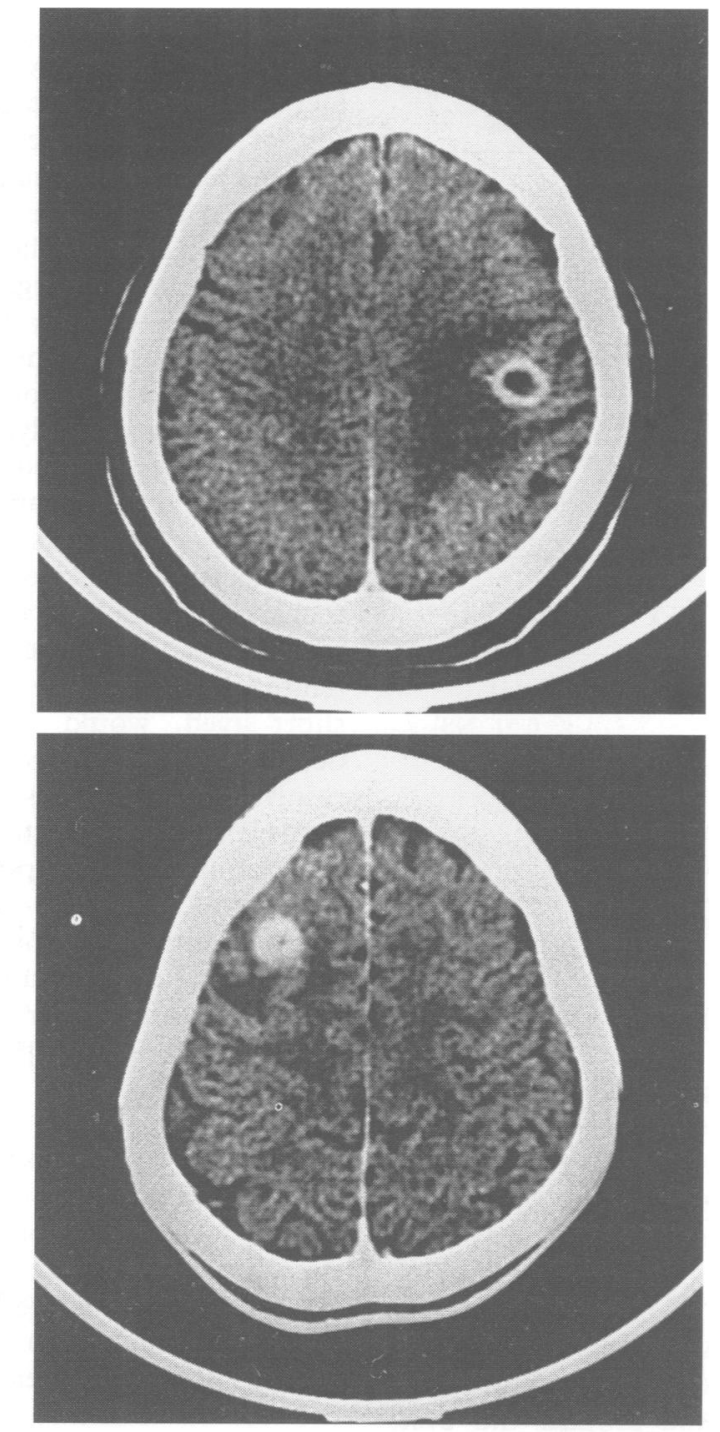

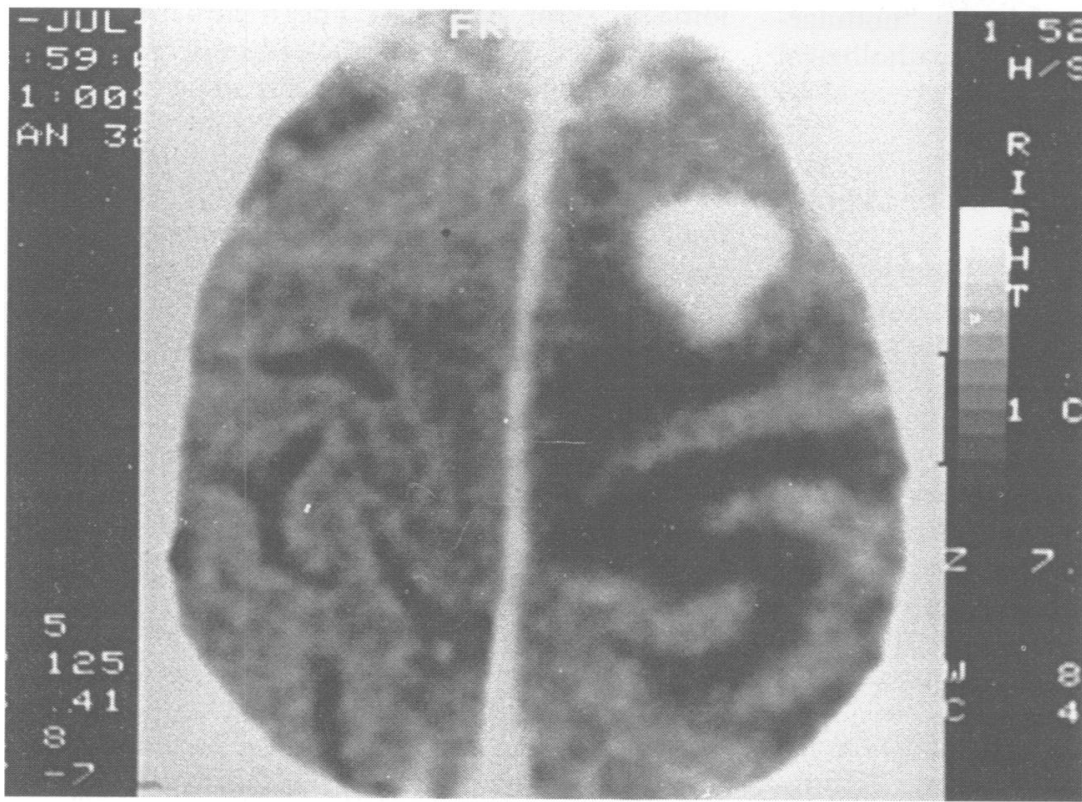

C described earlier. ${ }^{3}$ Type A lesions comprised solitary discs and rings, Type B lesions consisted of nodular lesions with two confluent discs or rings or a combination (figure). The oedema on CT was graded subjectively on a scale of 0 to 3 , grade 0 denoting no oedema and grade 3 severe oedema with a shift of the midline structures.
PROCESSING OF SAMPLES

The histological specimen (stained with haematoxylin and eosin) was examined for the presence of the parasite and neutrophils in the inflammatory reaction. If the specimen failed to disclose the parasite, multiple $5 \mu$ thick deeper sections were cut and stained for acid fast bacilli with Ziehl Neelsen stain and for fungal elements with periodic acid Schiff's and Gomori's methanamine stain.

The parasite was considered to be present in the granuloma when the whole parasite or parts such as the sucker, hooklets, or the outer membrane were seen.

\section{CORRELATIONS SOUGHT AND HYPOTHESES}

TESTED

In seeking correlations between the clinical and CT features and the pathological findings we formulated four hypotheses-namely: (1) the parasite was more likely to be seen in the granuloma of a patient with a shorter duration of symptoms (seizures) (< six months $v \geqslant \operatorname{six}$ months); (2) the parasite was more likely to be present in a "ring" type of CT lesion than other types of CT lesions ("disc" or type B); (3) the severity of the perilesional oedema on CT would be greater in female than in male patients; and (4) the presence of a neutrophilic response in the granuloma wall would be associated with a greater degree of oedema around the enhancing CT lesion.

\section{STATISTICAL METHODS}

A $\chi^{2}$ test was used to determine the statistical significance of differences. A P value $<0.05$ was considered significant.

\section{Results}

PATHOLOGICAL FINDINGS

Microscopic examination disclosed cavitary lesions containing parts of an intact or degenerate cysticercus in 22 , inflammatory cavitary lesions without the parasite in 13, and noncavitary hyalinised fibrous nodules with inflammation in eight patients. Of the 22 specimens with the cysticercus, only two had the entire parasite. In five patients degenerated forms of the cysticercus were seen, whereas in the rest (15) only parts of the parasite were visible.

An inflammatory response consisting of lymphocytes, plasma cells, and macrophages was present in all the granulomas. A neutrophilic infiltrate was, however, seen in only 10 of 22 specimens with the parasite and in nine without the parasite.

\section{CLINICORADIOLOGICAL AND PATHOLOGICAL} CORRELATIONS

Tables 1 and 2 summarise the results of the study. There was no significant correlation between the duration of symptoms (seizures) and the presence of the parasite in the granuloma. Similarly, the type of CT lesion was not correlated with the parasite presence. Finally, the presence of neutrophils in the granulomatous inflammation or the sex of the patient did not correlate with the degree of oedema around the lesion. 
Table 1 Duration of seizures, type of CT lesion, and parasite presence

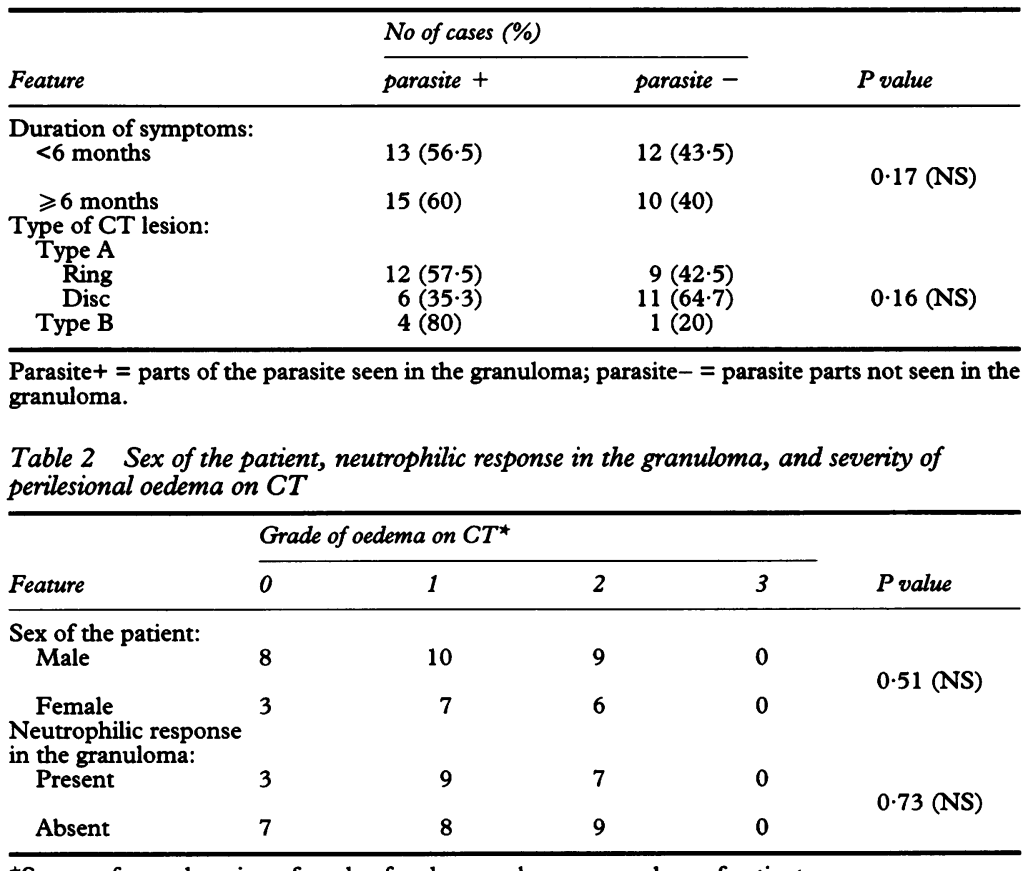

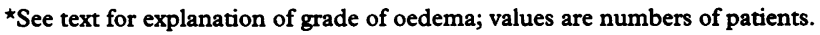

\section{Discussion}

PATHOLOGY OF PARENCHYMAL CEREBRAL CYSTICERCUS CYSTS

Cerebral cysticercosis is caused by the larval form of the parasite Taenia solium or the pork tapeworm. The live parasite does not generally produce any symptoms. The process of involution usually begins spontaneously with the disintegration of the outer vesicular membrane and discoloration of the fluid contents. This process also often heralds the onset of neurological symptoms. The inflammatory tissue forms a granuloma around the cyst, the cysticercus granuloma, or the "nodulargranular" stage in Escobar's pathological staging of the evolution of a cerebral parenchymal cysticercus cyst. ${ }^{6}$

DURATION OF SYMPTOMS, TYPE OF CT LESION, AND PARASITE PRESENCE

Although we did not expect all lesions producing symptoms for less than six months to have the parasite, we anticipated that a greater proportion of these lesions would have the parasite. The death of the parasite and degeneration of its contents leading to its disappearance from the granuloma was expected to be related to the time after the process of involution began, which closely precedes the onset of seizures. It is apparent that the natural history of the solitary cerebral cysticercus granuloma is extremely varied and is unpredictable in any given patient.

The second hypothesis was made on the premise that a cavitary lesion ("ring" lesion), which closely resembles the live larval form, is more likely to contain the scolex than a solid lesion ("disc" lesion); however, a high proportion of even apparently solid lesions on the CT ("discs") contained the parasite.

As CT was performed only about two hours before the granuloma was excised, any correlation that was to exist between the CT findings and the pathological attributes of the granuloma should have been reflected in our study.
HOST FACTORS DETERMINING INTENSITY OF PERILESIONAL OEDEMA

Del Brutto et $a l^{7}$ have shown a greater intensity of host response and hence degree of oedema around parenchymal cysticercus cysts in women than in men with the disease. We did not find any correlation between the sex of the patient and the degree of oedema.

We chose to study the effect of the neutrophilic response rather than the lymphocytic and plasma cell response on the perilesional oedema because the lymphocytic and plasma cell responses are constant features of all cysticercus granulomas including those which are in the end stages of their activity. Thus whereas these cells were seen in all our specimens, a neutrophilic response was only seen in 19 cases. Therefore, we presumed that the neutrophilic response, which is episodic and short lived, would correlate well with the oedema, which also occurs episodically and resolves spontaneously. Obviously factors derived from the neutrophils are not a major substrate for oedema production. The oedema is possibly linked to some humoral factors released either by the parasite or resulting from the host immune response but not dependent on the neutrophilic component of the inflammatory response or the sex of the patient.

IMPLICATIONS FOR CYSTICIDAL TREATMENT The cysticidal drugs albendazole and praziquantel act directly on the parasite. Whereas praziquantel produces paralysis of the cysticercal musculature, albendazole inhibits the uptake of glucose by the parasite's membranes. ${ }^{8}$ Cysticidal treatment possibly hastens the resolution of a solitary cysticercus granuloma in some patients. ${ }^{5}$ The treatment also confirms the diagnosis in the responders.

We postulated that if the presence of the parasite could be predicted on the basis of clinical or CT features then such a subgroup of patients might benefit from cysticidal treatment. Although there is no evidence to suggest that the presence of the parasite determines the response of the lesion to albendazole treatment, it seems a reasonable assumption to make as the drug acts on the parasite itself.

1 Rajshekhar V. Etiology and management of single smal CT lesions in patients with seizures: understanding a controversy. Acta Neurol Scand 1991;84:465-70.

2 Chandy MJ, Rajshekhar V, Ghosh S, et al. Single small enhancing CT lesions in Indian patients with epilepsy: clinical, radiological, and pathological considerations. Neurol Neurosurg Psychiatry 1991;54:702-5.

3 Rajshekhar V, Haran RP, Prakash GS, Chandy MJ. Differentiating solitary small cysticercus granulomas and tuberculomas in patients with epilepsy: clinical and computerized tomographic criteria. $\mathcal{F}$ Neurosurg 1993;78: puterized.

4 Rajshekhar V, Chandy MJ. Enlarging solitary cysticercus granulomas. $\mathcal{F}$ Neurosurg 1994;80:840-3.

5 Rajshekhar V. Albendazole therapy for persistent solitary cysticercus granulomas in patients with epilepsy. Neurology 1993;43:1238-40.

6 Escobar A. The pathology of neurocysticercosis. In: Palacios E, Rodriguez-Carabajal I, Taveras J, eds. Cysticercosis of the central nervous system. Springfield, Ill CC Thomas, 1983:27-54.

7 Del Brutto OH, Garcia E, Talamas O, Sotelo J. Sexrelated severity of inflammation in parenchymal brain cysticercosis. Arch Intern Med 1988;148:544-6.

8 Del Brutto OH, Sotelo J, Aguirre R, Diaz-Calderon E Alarcon TA. Albendazole therapy for giant subarachnoid cysticerci. Arch Neurol 1992;49:535-8. 